\title{
Clinician Perceptions About a Decision Support System to Identify and Manage Opioid Use Disorder
}

\author{
Leif I. Solberg, MD, Stephanie A. Hooker, PhD, MPH, Rebecca C. Rossom, MD, MS, \\ Anna Bergdall, MPH, and Benjamin F. Crabtree, PhD
}

Background: Addressing the opioid epidemic would benefit from primary care clinicians identifying and managing opioid use disorder (OUD) during routine clinical encounters, but current rates are low. Clinical decision support (CDS) systems are a promising way to facilitate such interactions, but will clinicians use them?

Methods: We iteratively conducted semi-structured interviews with 8 purposively sampled primary care clinicians participating in a pilot OUD-CDS study to identify attitudes toward discussing OUD and preferences for support in doing so. Five of them had used a pilot version of the CDS for 6 months, while the others were in comparison clinics. Interviews were recorded, transcribed, and analyzed by a multi-disciplinary group of experienced researchers, using an editing organizing style where the analysts independently highlighted relevant text and then discussed to reach a consensus on themes.

Results: We identified five themes: 1. Primary care is the right place to address 0UD. 2. Both clinicianpatient and clinician-clinician relationships affect how and whether clinicians address OUD in a particular patient encounter. 3. The main challenges are limited time and competing priorities for these complex patients. 4. Although a CDS for OUD could be very helpful, it must meet different needs for different clinicians and clinical situations and be simple to use. 5. For optimal benefit, the CDS needs to be complemented by supportive organizational policies and systems as well as local clinician encouragement.

Conclusions: With the right design and a supportive organization, these primary care clinicians believe a CDS could help them regularly identify and address OUD among their patients as long as it incorporates their concerns about relationships, competing priorities, patient complexity, and user simplicity. (J Am Board Fam Med 2021;34:1096-1102.)

Keywords: Attitude of Health Personnel, Clinical Decision-Making, Clinical Decision Support Systems, Opioid-Related Disorders, Opioids, Primary Health Care

\section{Introduction}

Opioid use disorder (OUD) has become the leading cause of accidental death in the United States. There were more than 3 times as many overdose deaths in 2016 compared with 1999, and age-

This article was externally peer reviewed.

Submitted 25 March 2021; revised 2 June 2021; accepted 4 June 2021.

From the HealthPartners Institute, Minneapolis, MN (LIS, SAH, RCR, AB); Rutgers Robert Wood Johnson Medical School, New Brunswick, NJ (BFC).

Funding: This work was supported by the National Institute on Drug Abuse's Clinical Trials Network. The views and opinions expressed in this manuscript are those of the authors only and do not necessarily represent the views, official policy, or position of the US Department of Health and Human Services or any of its affiliated institutions or agencies.

Conflict of interests: The authors have no conflicts of interest to declare.

Corresponding author: Leif I. Solberg, MD, HealthPartners Institute, 817033 rd Ave So., Bloomington, MN 55425, Phone: 952-967-5017 (E-mail: Leif.I.Solberg@HealthPartners.com). adjusted death rates are much higher in rural areas and certain states. ${ }^{1}$ Illicit drug use is defined by the National Survey on Drug Use and Health as both use of illegal drugs and misuse of prescription drugs. $^{2}$ In 2018, an estimated 1.6 million adults aged 26 years or older had an OUD in the previous year. ${ }^{2}$ Yet despite the frequency and seriousness of the problem, and even with several effective medications and therapies, relatively few people with OUD receive treatment. In an analysis of data from the National Survey, Creedon and Cook estimated that only 6 to $8 \%$ of people meeting criteria for past-year substance use disorders were receiving treatment, with little change from 2005 to $2014 .^{3}$

Because primary care clinicians most commonly provide opioid prescriptions, primary care is the most logical and feasible place to identify patients with or at risk for OUD and refer or initiate treatment for it. ${ }^{4,5}$ However, many studies have identified 
barriers to doing so, including time, competing priorities, risk of unpleasant reactions from patients, and lack of expertise. ${ }^{6-10}$

Most of these problems seem amenable to support from clinical decision support (CDS) built into the electronic health record; an approach demonstrated to improve care for various medical problems in the last decade. ${ }^{1-13}$ The Office of the National Coordinator for Health Information Technology describes CDS tools as providing "clinicians, staff, patients, or other individuals with knowledge and person-specific information, intelligently filtered or presented at appropriate times, to enhance health and health care." (https:// www.healthit.gov/topic/safety/clinical-decisionsupport) Spithoff et al's scoping review of CDS systems for opioid prescribing found some studies using this approach, but none included patient outcomes. ${ }^{14}$ However, a group at Ochsner Clinic demonstrated that the implementation of such a tool in 36 clinics greatly increased the number of patients receiving OUD therapy, and a group at the University of Texas Southwestern has been testing whether an OUD CDS can increase the initiation of buprenorphine treatment in emergency departments. ${ }^{15-18}$ A National Institute on Drug Abuse Center for the Clinical Trials Network (CTN) report in 2020 concluded that a CDS tool for OUD screening, assessment, and treatment might help address the opioid crisis. ${ }^{19}$

Our team has extensive experience with building and testing CDS systems and has been working on a version aimed at the CTN recommendations. However, in a preliminary field test with volunteer primary care clinicians, it was only used in $5 \%$ of the visits where patients were flagged as being at risk, even though $65 \%$ of participating physicians reported that they would recommend the OUD-CDS and found it helpful with screening, discussing, and prescribing for OUD. ${ }^{20}$ To better understand the potential value of a CDS, the reasons it was used so infrequently, and how primary care clinicians view their role in OUD, we conducted a series of semi-structured qualitative interviews with a sample of these clinicians.

\section{Methods}

The OUD-CDS tested in this study was designed to identify patients coming in for care who were at risk for OUD or had that diagnosis, alert the clinician with a banner in the electronic health record (EHR), and provide EHR tools to facilitate screening and diagnosis, treatment, and referral. It was designed to display for $8 \%$ of patient visits; a frequency considered feasible for busy clinicians. Data for this analysis were collected as part of a feasibility and acceptability pilot study conducted in the primary care clinics of a large multi-specialty care system in the metropolitan Minneapolis/St. Paul area of Minnesota. The care system has 1800 physicians with 600 in primary care and 190 advanced practice clinicians organized in 52 primary care clinics. The average patient panel is 1500 in primary care with 20 encounters per day, $25 \%$ of them virtual after the COVID-19 surge. A single EHR (Epic) is used by all health care personnel. Although there are some teaching rotations, most clinicians are in practice, and none of those interviewed supervise trainees.

Fifty-five primary care clinicians volunteered to participate in the pilot. Eight physicians were waivered to prescribe buprenorphine and were all assigned to the intervention arm. Of the non-waivered clinicians, 24 were randomized to the intervention and 23 to the control arms. For this study, we purposively sampled from these 3 groups of clinicians to ensure maximum diversity in leadership, intervention, and OUD care experience. All 10 clinicians we contacted agreed to participate, although only 8 could be scheduled.

The interviews were conducted at the interviewee's practice site or over the telephone by a family physician or psychiatrist, with a clinical psychologist or project manager as an observer. Interviews lasted 20 to 30 minutes and followed a guide that included 4 goals/grand tour question groupings:

1. Whether and how the CDS was helpful, what barriers existed to its use, and how it could be improved to better fit their needs (all were shown screenshots)?

2. What would be the most critical features of a helpful CDS?

3. How they viewed their role in addressing OUD with their patients?

4. How they approached patients about their OUD risk?

After the interview (usually the same day), a medical anthropologist, the interviewers, and 2 to 3 additional study team members gathered by conference call to hear a report from the attendees and ask questions or make comments. The main purposes were to identify important highlights immediately relevant to the ongoing revision of the CDS, create a structured summary of the interview before forgetting immediate impressions, and identify additional issues 
for subsequent interviews. By the sixth interview, it was clear from these immediate reviews that we were already approaching data saturation, so no additional interviews were scheduled after these initial $8 .^{21}$ Then, the interview recordings were professionally transcribed and de-identified.

Further analysis of the transcripts began with 3 study team members (LS, SH, BC) reading through each interview individually and highlighting segments of text of particular relevance to our aims. This activity was followed by a group meeting for each transcript to discuss the highlights and summarize the key observations from each interview. These observations were then organized by a group consensus process into a smaller number of themes from each interview and finally to a group consensus around the main themes and lessons across all the interviews. Each step was documented so the themes could be traced back to their origins. The study was reviewed, approved, and monitored by the HealthPartners Institutional Review Board.

\section{Results}

The interviewees included 2 waivered physicians and 1 who had become waivered after the pilot, 3 nonwaivered physicians, and 2 non-waivered physician assistants. Five were female and 3 male, 4 had roles as clinic or organizational leaders in addition to their clinical practice, and their clinical experience ranged from 3 to 26 years. Three had been in the control arm of the pilot, so they had not seen or used the CDS, while the other 5 had been in the intervention arm and used the CDS with 0,2, 4\%, 5\%, and $41 \%$ of eligible patient encounters when it identified a patient at possible OUD risk. On average, these intervention clinicians had 84 of 930 encounters where the CDS alert was displayed, including the clinician outlier (who used the CDS at $41 \%$ of eligible encounters). There was little apparent influence on Naltrexone use or referrals to specialty care for OUD, but non-waivered clinicians increased their rate of diagnosis of OUD by 11-fold, whereas those in the control clinics increased by 8 -fold.

In conducting the analyses, 5 main themes became apparent:

1. These clinicians agree that primary care is the right place to address OUD.

2. Both clinician-patient and clinician-clinician relationships affect how and whether they address OUD in a particular patient encounter.
3. Their main challenges are limited time and competing priorities for these complex patients.

4. Although a CDS for OUD could be very helpful, it must be designed to meet different needs for different clinicians and clinical situations and be simple to use.

5. For optimal benefit, the CDS needs to be complemented by supportive organizational policies and systems as well as local clinician encouragement.

\section{Primary Care is the Right Place to Address OUD}

All the clinicians agreed that OUD was their responsibility as primary care clinicians and that they were comfortable with bringing the topic up with patients (in the right situation), even when the patient had not raised the topic. When asked how he sees his role in addressing OUD, a non-waivered 14-year medical doctor said,

"Oh, buge. Huge. Very, very big-it starts with me, and it can be exacerbated by me-so my role is huge."

Similarly, a 3-year physician assistant said several times that "I think we have a big role." He also noted, "Like a lot of things in primary care, you know, we're often first line-I think we need to be a little bit more in tune about what we can do kind of as a primary provider for them and what we can offer them in terms of resources before they might get into the murky waters of outside referral, pain clinics."

A non-waivered 20-year medical doctor leader talked about the sentiment in her clinic among clinicians:

"We all absolutely see our role as being part of reducing, eliminating, tapping into resources, using chronic pain management clinic, looking at alternatives to narcotics."

She also said, "we are again increasingly confident in having discussions like that with the more difficult patients."

However, none of the interviewees felt that this was an exclusive role for primary care, recognizing that all specialties need to prescribe safely. Most should also take responsibility for the issue when they recognize it.

\section{Both Clinician-Patient and Clinician-Clinician Relationships Affect How and Whether They Address OUD in a Particular Patient Encounter}

Five of the interviewees spontaneously brought up the importance of relationships in dealing with OUD. They emphasized that these difficult discussions go so 
much better if there is a good pre-existing relationship with the patient that they are much less likely to bring up OUD when that is not present. A relatively recent physician assistant said,

"Hopefully, we establish a relationship with these patients, and we know them well enough that we can bring this up in a manner that they're receptive to it. The downside is, being [in] family practice, sometimes you get patients you've never seen before, and you look at their medication list, and they're coming in for more refills. I don't know if I would hesitate (to bring this up) as much as I would just have to be a little more cautious with my wording."

Patients who are new to them or are usual patients of another clinician are doubtful candidates for such discussion, not only because the conversation with the patient will be more challenging but because clinicians do not want to damage their relationship with the patient's usual clinician. A nonwaivered 15-year medical doctor leader with strong feelings about being firm with her own patients said,

"If 1 of my colleagues has started prescribing, I feel obligated to meet the promise or expectation of the patient. I have more of a feeling I need to support my colleague and trust in their decision-making in this. It doesn't mean we don't have those conversations offline, but it's not in front of the patient."

\section{Their Main Challenges Are Limited Time and Competing Priorities for These Complex Patients}

Six of the 8 clinicians identified their limited time with patients and all the competing priorities to pack into that time as making it challenging to add another topic, especially 1 as complex as OUD that often occurs in patients who are already medically and personally complex. One recent physician assistant dealt with that by stating,

"I would probably try it on select individuals or select situations where it wasn't going to be a lengthy visit, and I knew that I had some extra time to go through this."

The only 2 clinicians who did not raise this as a problem were 1 who only practiced 1 day a week and another who had uniquely high use rates which saw his role as more limited and focused entirely on sending patients to the pain clinic to wean them off narcotics. He also said the CDS helped him be efficient:

"It's easier for one to open up a topic when the patient is looking at the same screen - they realize that it's official, it's not just my dislike of opioids."
4. Although a CDS for OUD Could Be Very Helpful, It

Must Be Designed to Meet Different Needs for

Different Clinicians and Clinical Situations and

Must Be Simple to Use

Clinicians described various approaches and needs for discussing OUD with diverse patients and situations, so they all emphasized the need to design a CDS that could provide flexible support that was simple and easy to use. For example, waivered clinicians were looking for information about medication and treatment options and guidelines, both for themselves and patients.

Most non-waivered clinicians saw the value of the CDS to identify patients potentially at risk or as a way to facilitate referral to various specialty resources. Both waivered and non-waivered clinicians might use none or a few of the features briefly or in-depth, depending on the time available and complexity of that patient's needs and responses that day. Forcing a linear and protracted pattern of use would (and did) lead to low use levels. As a 26year, recently waivered medical doctor said,

"The more steps you make, the more clicks and the more complex, the higher the risk that people aren't going to use it. And so the simplicity of the tool is going to be really [important]."

One waivered 11-year medical doctor leader also wanted the CDS to facilitate documentation of what was discussed and done during the encounter, saying,

"Because I would need to document on paper what I was also documenting in the computer so that I could recreate the note. And so that was my main barrier to use."

\section{For Optimal Benefit, the CDS Needs to Be Complemented by Supportive Organizational Policies and Systems as Well as Local Clinician Encouragement}

Several interviewees suggested that taking on additional responsibility in busy primary care was only likely if the organization and clinician culture fully supported doing so, including both policies and guidelines as well as support from colleagues and staff. No matter how well designed, a CDS without such facilitation was unlikely to be used often by many clinicians. One specific example provided by a waivered 28-year medical doctor stating,

"We set something up with the ER [emergency room].

When they identify people that have come in and they can give first dose [of Suboxone] in the emergency 
room, and then can directly schedule a patient with me, and that [happened] once and it worked fabulous, and the patient has done really well."

Another example involved the schedule providing an early warning that the CDS had identified a visit with a potentially at-risk patient so the clinician and staff could prepare for the discussion.

A non-waivered 20-year medical doctor leader reflected on the need for supportive colleagues and culture saying,

"We have excellent commitment from our partners to be consistent about appropriate refills and using controlled-substance agreements, so I would say we have a robust practice to avoid, eliminate, reduce the use of narcotics.”

\section{Discussion}

These primary care clinicians all strongly supported the idea that it is their responsibility to address OUD, at least for patients with whom they have a good relationship and within the limitations of time pressure and competing priorities. They also all recognized the potential value of support from a CDS system that is connected to their EHR system, as long as it is simple to use and provides a variety of supports and resources for individualized situations and goals. Most also recognized that such a CDS would be most effective if it were actively supported by the organization and integrated with other relevant programs and resources. Although we cannot answer the question of whether clinicians will use a CDS to identify and manage OUD among their patients, they seemed to accept the need to do that, expressed interest in the support a well-designed and integrated CDS could provide, and named barriers for OUD discussions that a CDS might be able to help.

Clearly, the first iteration of this CDS did not meet these requirements since it was only used in $5 \%$ of the visits where it identified patients as being at risk for OUD. The information provided by these interviews led to an extensive redesign and testing effort that will hopefully result in much higher use and better support for what is recognized by these clinicians as a sensitive and important conversation with patients.

The recently revised recommendations from the United States Preventive Services Task Force include a recommendation to screen adults for unhealthy drug use by asking them questions about it (level $\mathrm{B}$ evidence). This recommendation has provided a new impetus for an effective CDS system for OUD, even though the report's Research Needs section does not explicitly mention a CDS system among its options. ${ }^{22}$ Almost simultaneously, an expert panel convened by the National Institute on Drug Abuse issued a report that emphasized that "Integration of OUD screening, assessment, and treatment within primary care systems could potentially help stem the tide of this epidemic." 19 The report described an "outline of clinical decision support that may facilitate this process," including incorporating algorithms in electronic health records and developing complementary workflow systems for OUD as identified by our interviewees in the fourth theme.

The barriers of time and competing priorities in primary care identified in the third theme are not new; they have been described and quantified for at least 30 years, initially concerning the desire for primary care practices to provide more preventive services, but now also voiced for substance use disorders. $^{7,23-26}$ Brooks et al also identified the high prevalence of medical, psychological, and social challenges among patients with OUD. ${ }^{27}$ These barriers come up again with each new societal problem assigned to medicine and health care, recently with social determinants of health and now the opioid crisis. ${ }^{28}$ Although the complaint is real and needs effective solutions, the solution usually lies in some combination of practice systems, expanded care team roles, and integration with specialty and community resources. ${ }^{29-32}$ Therefore, a CDS is a potentially important solution for these barriers.

For a CDS to be helpful, it will be important to address both the design features identified by these interviewees in theme 1 and the NIDA report, as well as the organizational support and workflow concerns identified in theme $2 .{ }^{19}$ Both Walley et al and Hutchinson et al's studies of primary care physician barriers to prescribing buprenorphine highlighted concerns about the lack of institutional support, especially from mental health and psychosocial services, as a cause of nonuse. ${ }^{7,9}$ A more sophisticated study of the overall value of organizational support came from Ike et al who demonstrated that both clinicians and staff reported improved work-life after implementing systems-based improvements for opioid medication management in their practices. ${ }^{33}$

Perhaps the most uniquely important information from these interviews comes from these clinicians' unanimous assertion of OUD being a prime 
responsibility for them as primary care clinicians and their corresponding recognition of the central importance of their relationships with both patients and fellow clinicians to their ability to fulfill that role well (themes 1 and 2). Saunders et al have confirmed that patients also see rapport with their clinicians as essential to screening for alcohol and drug use. ${ }^{34}$ Understanding this is essential to guideline developers and care systems as they put increasing pressure on clinicians to address OUD in their practices.

As we work to incorporate these important findings in our development and implementation of a CDS system throughout this care system, we are mindful of the limitations that they came from only 8 clinicians. Moreover, these clinicians had volunteered for an earlier pilot study of a CDS and these interviews, so they certainly may have a greater than average interest in both the problem of OUD and its solution. As we undertake future qualitative work to understand clinician perspectives on the next version of the CDS on a broader array of non-volunteers, we will be interested in obtaining a wider range of perspectives. We also recognize the need to assess this approach's effectiveness, costs, and patient/staff satisfaction. However, we mainly wanted more focused guidance and insights from those with greater interest and experience at this stage.

Iterative interviews can provide important lessons for a new and rapidly developing field like that of the opioid epidemic. If we can successfully incorporate the lessons from this study, future participants (both clinicians and patients) will benefit, and we may see primary care move from being a part of the problem to a key resource for its solution.

To see this article online, please go to: http://jabfm.org/content/ 34/6/1096. full.

\section{References}

1. Hedegaard H, Warner M, Minino AM. Drug overdose deaths in the United States, 1999-2016. NCHS Data Brief 2017;2017:1-8.

2. SAMHSA. Key substance use and mental bealth indicators in the United States: Results from the 2018 National Survey on Drug Use and Health. Rockville, MD, 2019.

3. Creedon TB, Cook BL. Access to mental health care increased but not for substance use, while disparities remain. Health Aff (Millwood) 2016;35:1017-21.

4. Chen JH, Humphreys K, Shah NH, Lembke A. Distribution of opioids by different types of Medicare prescribers. JAMA Intern Med 2016;176:259-61.
5. Dobscha SK, Morasco BJ, Duckart JP, Macey T, Deyo RA. Correlates of prescription opioid initiation and long-term opioid use in veterans with persistent pain. Clin J Pain 2013;29:102-8.

6. Barry DT, Irwin KS, Jones ES, et al. Integrating buprenorphine treatment into office-based practice: a qualitative study. J Gen Intern Med 2009;24:218-25.

7. Hutchinson E, Catlin M, Andrilla CH, Baldwin LM, Rosenblatt RA. Barriers to primary care physicians prescribing buprenorphine. Ann Fam Med 2014;12:128-33.

8. Netherland J, Botsko M, Egan JE, BHIVES Collaborative, et al. Factors affecting willingness to provide buprenorphine treatment. J Subst Abuse Treat 2009;36:244-51.

9. Walley AY, Alperen JK, Cheng DM, et al. Officebased management of opioid dependence with buprenorphine: clinical practices and barriers. J Gen Intern Med 2008;23:1393-8.

10. Huhn AS, Dunn KE. Why aren't physicians prescribing more buprenorphine? J Subst Abuse Treat 2017;78:1-7.

11. Kharbanda EO, Asche SE, Sinaiko A, et al. Evaluation of an electronic clinical decision support tool for incident elevated BP in adolescents. Acad Pediatr 2018;18:43-50.

12. O'Connor PJ, Sperl-Hillen JM. Current status and future directions for electronic point-of-care clinical decision support to improve diabetes management in primary care. Diabetes Technol Ther 2019;21:S226-S234.

13. Sperl-Hillen JM, Crain AL, Margolis KL, et al. Clinical decision support directed to primary care patients and providers reduces cardiovascular risk: a randomized trial. J Am Med Inform Assoc 2018; 25:1137-46.

14. Spithoff S, Mathieson S, Sullivan F, et al. Clinical decision support systems for opioid prescribing for chronic non-cancer pain in primary care: a scoping review. J Am Board Fam Med 2020;33:529-40.

15. Holland WC, Nath B, Li F, et al. Interrupted time series of user-centered clinical decision support implementation for emergency department-initiated buprenorphine for opioid use disorder. Acad Emerg Med 2020;27:753-63.

16. Melnick ER, Jeffery MM, Dziura JD, et al. User-centred clinical decision support to implement emergency department-initiated buprenorphine for opioid use disorder: protocol for the pragmatic group randomised EMBED trial. BMJ Open 2019;9:e028488.

17. Melnick ER, Nath B, Ahmed OM, et al. Progress report on EMBED: a pragmatic trial of user-centered clinical decision support to implement EMergency Department-Initiated BuprenorphinE for opioid use disorder. J Psychiatr Brain Sci 2020;5.

18. Price-Haywood EG, Robinson W, Harden-Barrios J, Burton J, Burstain T. Intelligent clinical decision 
support to improve safe opioid management of chronic noncancer pain in primary care. Ochsner J 2018;18:30-5.

19. Bart GB, Saxon A, Fiellin DA, et al. Developing a clinical decision support for opioid use disorders: a NIDA center for the clinical trials network working group report. Addict Sci Clin Pract 2020;15:4.

20. Rossom RC, Sperl-Hillen JM, O’Connor PJ, et al. Functionality and clinician acceptance of a clinical decision support tool to improve primary care of opioid use disorder. In preparation.

21. Saunders B, Sim J, Kingstone T, et al. Saturation in qualitative research: exploring its conceptualization and operationalization. Qual Quant 2018;52:1893-907.

22. Krist AH, Davidson KW, Mangione CM, USPSTF, et al. Screening for unhealthy drug use: US Preventive Services Task Force recommendation statement. JAMA 2020;323:2301-9.

23. Kottke TE, Brekke ML, Solberg LI. Making "time" for preventive services. Mayo Clin Proc 1993;68:785-91.

24. Yarnall KS, Pollak KI, Ostbye T, Krause KM, Michener JL. Primary care: is there enough time for prevention? Am J Public Health 2003;93:635-41.

25. Jaen CR, Stange KC, Nutting PA. Competing demands of primary care: a model for the delivery of clinical preventive services. J Fam Pract 1994;38: 166-71.

26. McNeely J, Kumar PC, Rieckmann T, et al. Barriers and facilitators affecting the implementation of substance use screening in primary care clinics: a qualitative study of patients, providers, and staff. Addict Sci Clin Pract 2018;13:8.
27. Brooks EM, Tong S. Implementing office-based opioid treatment models in primary care. J Am Board Fam Med 2020;33:512-20.

28. Solberg LI. Theory vs practice: should primary care practice take on social determinants of health now? No. Ann Fam Med 2016;14:102-3.

29. Bordley WC, Margolis PA, Stuart J, Lannon C, Keyes L. Improving preventive service delivery through office systems. Pediatrics 2001;108:E41.

30. Goodwin MA, Zyzanski SJ, Zronek S, et al. A clinical trial of tailored office systems for preventive service delivery. The Study to Enhance Prevention by Understanding Practice (STEP-UP). Am J Prev Med 2001;21:20-8.

31. Levengood TW, Peng Y, Xiong KZ, Community Preventive Services Task Force, et al. Team-based care to improve diabetes management: a community guide meta-analysis. Am J Prev Med 2019;57: e17-e26.

32. Jaen CR, McIlvain H, Pol L, Phillips RL, Jr., Flocke S, Crabtree BF. Tailoring tobacco counseling to the competing demands in the clinical encounter. J Fam Pract 2001;50:859-63.

33. Ike B, Baldwin LM, Sutton S, Van Borkulo N, Packer C, Parchman ML. Staff and clinician worklife perceptions after implementing systems-based improvements to opioid management. J Am Board Fam Med 2019;32:715-23.

34. Saunders EC, Moore SK, Gardner T, et al. Screening for substance use in rural primary care: a qualitative study of providers and patients. J Gen Intern Med 2019;34:2824-32. 